\title{
Monitoring resistance to Bacillus thuringiensis subsp. israelensis in the field by performing bioassays with each Cry toxin separately
}

\author{
Guillaume Tetreau ${ }^{+}$, Renaud Stalinski, Jean-Philippe David, Laurence Després \\ Laboratoire d'Ecologie Alpine, Unité Mixte de Recherche 5553, Université de Grenoble 1, Grenoble, France
}

\begin{abstract}
Bacillus thuringiensis subsp. israelensis (Bti) is increasingly used worldwide for mosquito control and is the only larvicide used in the French Rhône-Alpes region since decades. The artificial selection of mosquitoes with fieldpersistent $\mathrm{Bti}$ collected in breeding sites from this region led to a moderate level of resistance to Bti, but to relatively high levels of resistance to individual Bti Cry toxins. Based on this observation, we developed a bioassay procedure using each Bti Cry toxin separately to detect cryptic Bti-resistance evolving in field mosquito populations. Although no resistance to Bti was detected in none of the three mosquito species tested (Aedes rusticus, Aedes sticticus and Aedes vexans), an increased tolerance to Cry4Aa (3.5-fold) and Cryl1Aa toxins (8-fold) was found in one Ae. sticticus population compared to other populations of the same species, suggesting that resistance to Bti may be arising in this population. This study confirms previous works showing a lack of Bti resistance in field mosquito populations treated for decades with this bioinsecticide. It also provides a first panorama of their susceptibility status to individual Bti Cry toxins. In combination with bioassays with Bti, bioassays with separate Cry toxins allow a more sensitive monitoring of Bti-resistance in the field.
\end{abstract}

Key words: Bacillus thuringiensis subsp. israelensis - bioassays - field resistance - Cry toxins - mosquito

Numerous insect species represent major economic and sanitary issues for human societies as they can strongly impact crop cultures or transmit severe human diseases such as malaria, leishmaniasis or trypanosomiasis (Medlock et al. 2012, Savary et al. 2012, van Griensven \& Diro 2012). The control of pest populations is therefore of high importance and relies mainly on the use of chemical and biological insecticides (Meissle et al. 2011, van den Berg 2011). The main threat for an efficient pest control is the ability of insects to quickly develop resistance to nearly all the insecticides used, leading to a strong decrease of insecticides efficiency (Tabashnik et al. 2008, Rivero et al. 2010). To bypass these resistances and adapt the insecticide treatment strategies, detecting incipient resistance in treated populations as early as possible is required (Carriere et al. 2010).

The most obvious approach to detect resistance in the environment is to perform bioassays on field-sampled individuals, which consist in exposing insects to increasing amount of insecticide to calculate their relative tolerance to this insecticide (Sivasupramaniam et al. 2007). Such basic approach is often preliminary to

\footnotetext{
doi: 10.1590/0074-0276130155

Financial support: ANR (ANR-08-CES-006-01 DIBBECO)

GT was supported by the French Ministry of Research and RS was supported by the French Rhône-Alpes region.

GT current address: Department of Entomology, Cornell University,

New York State Agricultural Experiment Station, Geneva, NY, USA

+ Corresponding author: guillaume.tetreau@gmail.com

Received 14 January 2013

Accepted 10 April 2013
}

further studies exploring in-depth the molecular bases of the resistance (Tabashnik et al. 2006, Marcombe et al. 2009). Bioassays have been performed on numerous field populations of insects with different chemical insecticides (Ocampo et al. 2011, Shin et al. 2011, Silva et al. 2011, Springate \& Colvin 2012), but also with biological insecticides such as Bacillus sphaericus (Su \& Mulla 2004, Silva et al. 2008, Akiner et al. 2009) and Bacillus thuringiensis subspecies (Tabashnik et al. 1990, Gao et al. 2010, Tabashnik \& Carriere 2010, Alcantara et al. 2011, Gassmann et al. 2011). B. thuringiensis subsp. israelensis (Bti) is increasingly used worldwide for mosquito control and investigating potential Bti-resistance in the environment is essential to ensure the long term use of this insecticide. Resistance to Bti has been extensively searched in natural populations of mosquitoes (Liu et al. 2004, Vasquez et al. 2009, Loke et al. 2010, Kamgang et al. 2011) and generally concluded in a lack of $B t i$ resistance in field populations even after decades of Bti treatments, although a few studies described a decreased susceptibility in some populations (Zhang et al. 2004, Paul et al. 2005, Boyer et al. 2007, 2012). Nevertheless, these studies may suffer from technical biases, notably on the choice of the reference strain.

Usually, when bioassays are performed, a laboratory strain from the same species, susceptible to the insecticide tested, is used as a reference to calculate the resistance ratio of the strains/populations tested. Although this approach is widely used, it is adapted only if the reference strain has been sampled recently in populations geographically close to those tested. If this is not the case, results can reflect a basal difference of tolerance between the field populations and the reference strain, independent from Bti-treatments, rather than a selected resistance evolving in the populations treated with $B t i$ 
(Zhang et al. 2004, Paul et al. 2005). Moreover, this approach is only possible for a restricted number of species, as most mosquito species cannot be maintained in laboratory conditions. An alternative solution is to compare treated and untreated populations; but even in this case, the differences observed can be due to natural tolerance differences between populations and not to a selected resistance (Boyer et al. 2007, 2012). Therefore when mosquitoes cannot be maintained in laboratory, the only way of detecting incipient resistance is to apply a highly sensitive bioassay approach on field-populations and test for the evolution of their tolerance for several years (Janmaat \& Myers 2003, Tabashnik \& Carriere 2010). Strong evidence for an evolved resistance in the environment is given by an increasing tolerance to the insecticide in the same population over generations. Unfortunately, one of the main reasons why no firm resistance to $B t i$ was detected in the field yet may be due to the low resolution of bioassays performed with Bti, a mixture of four main toxins (Cry4Aa, Cry4Ba, Cry11Aa and Cyt1Aa).

Recent works on Bti-resistance showed that while levels of resistance to Bti can be low in a laboratory-selected strain (3.5-fold), resistance to each of the Cry toxins tested separately in bioassays can be much higher (up to 60-fold) (Paris et al. 2011, Tetreau et al. 2012b). Here we present an improved bioassay method consisting in performing distinct bioassays with each of the three $B t i$ Cry toxins on field populations of mosquitoes. We applied this approach to various mosquito populations collected in the French Rhône-Alpes region, where Bti is the only larvicide used for mosquito control since 1990. Furthermore, the leaf litters containing field-persistent $B t i$ able to select for resistance in mosquitoes in the laboratory were sampled in this region, raising concerns about possible Bti-resistance evolving in mosquitoes in these particular breeding sites (Tilquin et al. 2008, Paris et al. 2010, 2011). The variations in susceptibilities of field mosquito populations to Bti and Cry toxins are described and discussed. Perspectives of further improvement and wide application of this tool are also discussed.

\section{MATERIALS AND METHODS}

Mosquito sampling and species identification - Mosquito larvae were sampled in 17 different breeding sites of the French Rhône-Alpes region in close collaboration with the Entente Interdépartementale pour la Démoustication (EID) (Fig. 1). Among them, 12 sites were regularly treated with $B t i$ for decades and five had never been treated with Bti. Each sampling site is identified by the letter "P" followed by a number. Each site can be associated to different "P" numbers corresponding to different sampling dates. As mosquito breeding sites often contained more than one mosquito species, different species sampled at the same time in the same site were associated to the same "P" number.

As numerous different species can share the same breeding site, larvae were individually identified before bioassays using the identification key provided in Becker et al. (2010). Bioassays with different species were performed independently.
Production of individual Bti Cry toxins - A crystal negative strain of $B t i$ (4Q2-81) transformed with the plasmids pHT606, pHT618 or pWF53 was used for the production of Cry4Aa, Cry4Ba or Cryl1Aa toxins respectively. These strains were obtained from the Pasteur Institute (Paris, France) or from Prof. B Federici (University of California, Riverside, CA, USA). Each toxin was produced, purified and quantified as previously described (Tetreau et al. 2012b). Toxin suspensions were stored at $-20^{\circ} \mathrm{C}$ until use.

Bioassays procedure and data analyses - To avoid any influence of the larval stage on bioassays, larvae were maintained in the water from the breeding site where they were sampled until the third instar. Considering that the species studied cannot reproduce in laboratory conditions, we could not perform bioassays on F1 larvae, but directly used field-collected larvae. In order to limit the impact of environmental parameters in the sampled sites (e.g. pH of the water, pollutants etc.) on bioassay results, field collected larvae were kept in fresh water for $24 \mathrm{~h}$ before performing the bioassays.

When more than 2,000 larvae of the same species were sampled, standard bioassays were conducted by exposing larvae to five-seven concentrations of Cry4Aa, Cry4Ba, Cry11Aa and commercial Bti (Vectobac WG, $3500 \mathrm{ITU} / \mathrm{mg}$ ) for $48 \mathrm{~h}$ in order to obtain $5-95 \%$ mortality. When 1,000-2,000 larvae were sampled, high sensitive standard bioassays could be performed, but not for all the Cry toxins; bioassays with Bti and Cry4Aa were performed in priority since Cry4Aa is the main toxin persisting in the field after Bti treatment (Tetreau et al. 2012a) and with the highest resistance ratio in the Bti-

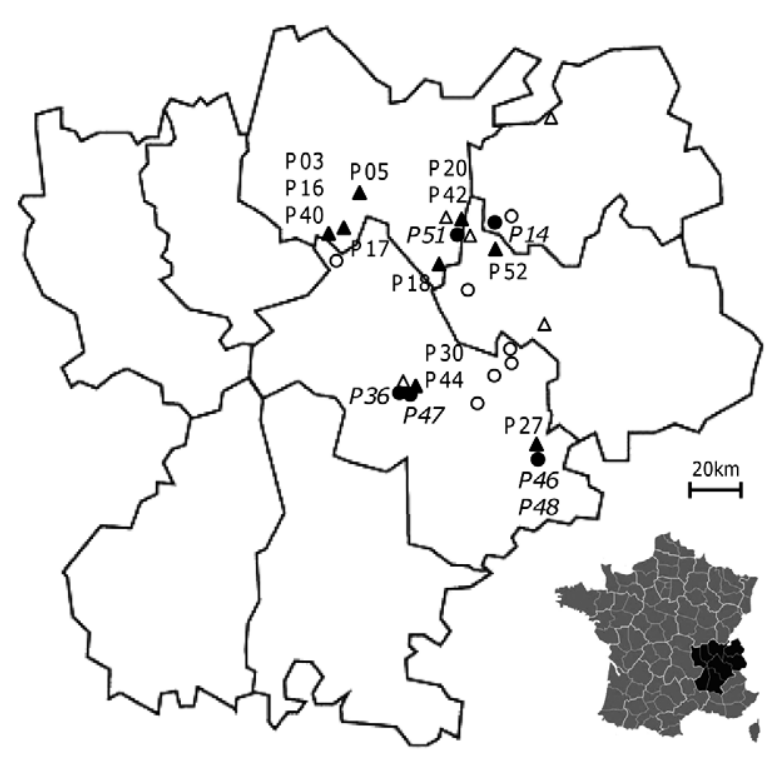

Fig. 1: localisation of the 32 mosquito breeding sites visited in the French Rhône-Alpes region. The 22 treated (triangles) and the 10 untreated (circles) sites visited are shown. Sampled sites used for bioassays ( 12 for treated and 5 for untreated sites) are indicated by a filled item and by the name of the population in italic (untreated site) or not (treated site). 
resistant strain described in Tetreau et al. (2012b). Lethal concentrations for $50 \%$ individuals $\left(\mathrm{LC}_{50}\right)$ were calculated for each population and each toxin using a probit statistical model with the module "dose" of XLSTAT v.2009.4.06 (Addinsoft).

When only a limited number of larvae of the same species were sampled, standard bioassays could not be performed, as a reduced number of replicates and/or concentrations performed would lead to a high variability in larval mortality and to a low reliability of the $\mathrm{LC}_{50}$ values. In this case, a "diagnostic dose" approach was used, consisting in using one concentration of Cry toxin or Bti chosen in order to obtain $60-80 \%$ mortality. These doses were designed for each species and each toxin from the bioassays data from the first population sampled. For each toxin and Bti, statistical differences in larval mortality between two populations from the same species were measured by a Wilcoxon test performed with $\mathrm{R}$ 2.8.1 software (R Development Core Team 2007).

For each toxin tested, four replicates per concentration was performed on 20 third-instar larvae in $50 \mathrm{~mL}$ of tap water containing the insecticide according to the standard bioassay procedure described by the World Health Organization (WHO 2005). For each population tested, five negative controls, consisting in 20 larvae exposed to tap water only, were performed. All bioassays were performed at $17^{\circ} \mathrm{C}$. For each population, the mean larval size was calculated from more than 15 larvae.

Significance of larval size and mortality differences between the three species (Aedes rusticus, Aedes sticticus and Aedes vexans) were estimated by performing a Wilcoxon test using R 2.8.1 software (R Development Core Team 2007).

Considering that the species studied cannot be maintained in laboratory, we were unable to compare the tolerance levels of the sampled populations to a susceptible laboratory strain. Therefore, for each species we com- pared populations of mosquitoes with each other. Then, we calculated "tolerance ratios" which represent an increased or decreased susceptibility of a population relative to the others.

\section{RESULTS AND DISCUSSION}

Limits of performing bioassays on field populations - Among the 71 natural mosquito populations sampled, data from 59 populations ( $83 \%$ ) were not analysable due to insufficient larvae to perform bioassays $(69 \%)$, to mortality in controls $(11 \%)$ or to high rate of pupation during bioassays (3\%) (Table I). No bioassay data could be obtained for Aedes cantans, Aedes cataphylla, Aedes cinereus and Culex pipiens field populations in the present study, while sufficient data were obtained for Ae. rusticus, Ae. sticticus and Ae. vexans populations (Table I). Among the three Ae. rusticus populations tested in bioassays, two were sampled in treated sites (P3 and P5) and one in an untreated site (P14) while Ae. sticticus (P16, P20 and P52) and Ae. vexans (P18, $\mathrm{P} 20, \mathrm{P} 30, \mathrm{P} 42$ and $\mathrm{P} 44)$ populations were all sampled in treated sites. Only one population of Culiseta annulata (P51) was sampled in sufficient amount to perform a diagnostic dose approach and showed comparable mortality rates as Ae. vexans populations at the same doses (data not shown).

The fact that only less than $17 \%$ of the populations sampled and only four out of the eight species sampled gave analysable results highlights the difficulty to find sites producing enough calibrated larvae of the same species to perform bioassays. This limit is mainly due to typical features of the Rhône-Alpes region, constituted of numerous sites of moderate size largely scattered in woodlands.

Interspecific comparisons - Ae. sticticus and Ae. vexans larvae exhibited the same patterns of tolerance (Wilcoxon test, $\mathrm{p}>0.05$ ) except for Cry4Aa for which

TABLE I

Summary of mosquito breeding sites sampled where at least one of the eight studied species were found

\begin{tabular}{|c|c|c|c|c|c|c|}
\hline \multirow[b]{2}{*}{ Species } & \multirow[b]{2}{*}{ Sampled } & \multicolumn{2}{|c|}{ Bioassays } & \multicolumn{3}{|c|}{ Not analysable populations } \\
\hline & & $\mathrm{LC}_{50}$ & $\begin{array}{l}\text { Diagnostic } \\
\text { dose }\end{array}$ & $\begin{array}{c}\text { Low } \\
\text { sample size }\end{array}$ & $\begin{array}{l}\text { High mortality } \\
\text { in controls }\end{array}$ & Pupation \\
\hline Aedes cantans & 11 & 0 & 0 & 9 & 2 & 0 \\
\hline Aedes cataphylla & 5 & 0 & 0 & 5 & 0 & 0 \\
\hline Aedes cinereus & 3 & 0 & 0 & 1 & 1 & 1 \\
\hline Aedes rusticus & 15 & 3 & 0 & 12 & 0 & 0 \\
\hline Aedes sticticus & 14 & 3 & 0 & 8 & 3 & 0 \\
\hline Aedes vexans & 12 & 3 & 2 & 6 & 0 & 1 \\
\hline Culex pipiens & 6 & 0 & 0 & 4 & 2 & 0 \\
\hline Culiseta annulata & 5 & 0 & 1 & 4 & 0 & 0 \\
\hline Total & 71 & 9 & 3 & 49 & 8 & 2 \\
\hline
\end{tabular}

for each species, the number of populations for which bioassays were performed is indicated. The main reason why bioassays were not performed for the other populations is also indicated. $\mathrm{LC}_{50}$ : lethal concentrations for $50 \%$ individuals. 
TABLE II

Mean lethal concentrations for $50 \%$ individuals $\left(\mathrm{LC}_{50}\right)$ calculated for all the populations of each species and tolerance ratios obtained for each species with individual Cry toxins and commercial Bacillus thuringiensis subsp. israelensis (Bti)

\begin{tabular}{lccccc}
\hline & & \multicolumn{3}{c}{ LC $_{50}$} \\
\cline { 3 - 6 } Species & $\begin{array}{c}\text { Size } \\
(\mathrm{mm})\end{array}$ & $\begin{array}{c}\text { Cry4Aa } \\
(\mathrm{ng} / \mathrm{mL})\end{array}$ & $\begin{array}{c}\text { Cry4Ba } \\
(\mathrm{ng} / \mathrm{mL})\end{array}$ & $\begin{array}{c}\text { Cry11Aa } \\
(\mathrm{ng} / \mathrm{mL})\end{array}$ & $\begin{array}{c}B_{t i}{ }^{a} \\
(\mu \mathrm{g} / \mathrm{mL})\end{array}$ \\
\hline Aedes rusticus & $10.6 \pm 0.8$ & $262.7 \pm 88.3$ & $60.9 \pm 15.2$ & $106.9 \pm 22.2$ & $2.03 \pm 1.05$ \\
Aedes sticticus & $6.3 \pm 0.9$ & $14.8 \pm 6.8$ & $8.9 \pm 0.0$ & $15.7 \pm 11.0$ & $0.11 \pm 0.028$ \\
Aedes vexans & $6.2 \pm 0.6$ & $67.6 \pm 6.4$ & $13.5 \pm 0.4$ & $27.1 \pm 11.7$ & $0.11 \pm 0.009$ \\
\hline
\end{tabular}

Tolerance ratios

\begin{tabular}{lccccc}
\cline { 3 - 5 } Ratio sp1/sp2 & Size & Cry4Aa & Cry4Ba & Cry11Aa & $B^{a}$ \\
\hline Ae. rusticus/Ae. sticticus & $1.7 \mathrm{x}$ & $17.8 \mathrm{x}$ & $6.8 \mathrm{x}$ & $6.8 \mathrm{x}$ & $18.5 \mathrm{x}$ \\
Ae. rusticus/Ae.vexans & $1.7 \mathrm{x}$ & $3.9 \mathrm{x}$ & $4.5 \mathrm{x}$ & $3.9 \mathrm{x}$ & $18.5 \mathrm{x}$ \\
Ae. vexans/Ae. sticticus & $1.0 \mathrm{x}$ & $4.6 \mathrm{x}$ & $1.5 \mathrm{x}$ & $1.7 \mathrm{x}$ & $1.0 \mathrm{x}$ \\
\hline
\end{tabular}

$a$ : Vectobac WG. Larval size ratios between species are also indicated.

Ae. vexans larvae were 4.6-fold more tolerant than $A$. sticticus larvae (Wilcoxon test, $\mathrm{p}<0.05$ ) (Table II). Ae. rusticus was significantly 3.9-fold-18.5-fold more tolerant than Ae. sticticus and Ae. vexans species when tested with Cry toxins and commercial Bti (Wilcoxon test, $\mathrm{p}<$ 0.05) (Table II). Ae. sticticus and Ae. vexans had similar larval size (6.3 and $6.2 \mathrm{~mm}$; Wilcoxon test, p > 0.05) while Ae. rusticus larvae were significantly $70 \%$ larger than larvae from other species $(10.6 \mathrm{~mm}$; Wilcoxon test, $\mathrm{p}<0.05$ ) (Table II). Correlating larval size with Bti toxins tolerance indicated that the higher tolerance of $\mathrm{Ae}$. rusticus larvae compared to the two other species is due to the size differences (Supplementary data), linked to its particular life cycle containing a long winter larval period. This induces a slow larval development and metabolism that could lead to an alteration of feeding behaviour, therefore reducing the quantity of Bti ingested that could partly explain the lower susceptibility to Bti observed for this species. These results are congruent with observations made by the local mosquito control agency EID that Ae. rusticus requires a higher dose of $B t i$ than other Aedes species for efficient control.

Intraspecific comparisons - Among the populations analysed, no increased tolerance to Bti was found in $A e$. rusticus, Ae. sticticus and Ae. vexans (Figs 2-5). This result is congruent with the observations of the EID who reported no decreased efficacy of Bti treatments in the treated sites over time and also with previous works conducted in other Bti-treated areas (Liu et al. 2004, Vasquez et al. 2009, Loke et al. 2010, Kamgang et al. 2011).

The untreated P14 population of Ae. rusticus was significantly 1.77 -fold more tolerant than treated P3 and P5 populations to Cry4Aa, but 1.72-fold less tolerant than P5 to Cryl1Aa (Fig. 2). No significant differences be- tween these populations were found when tested with Cry4Ba and Bti (Fig. 2).

The tolerance of Ae. vexans populations for which full bioassays were performed did not show significant variations for Cry4Aa, Cry4Ba and Bti while the P44 population was 2.5 -fold more tolerant than the P20 population to Cry11Aa (Fig. 3). P30 and P42 populations, for which the diagnostic doses approach was used, did not show any significant differential tolerance to Cry4Ba, Cryl1Aa and Bti (Fig. 4).

Bioassays with individual Cry toxins being more sensitive than bioassays with the whole Bti toxins mixture, slight changes in tolerance were observed to Cry4Aa and Cry11Aa in the P14 population of Ae. rusticus and to Cry11Aa in the P44 population of Ae. vexans.

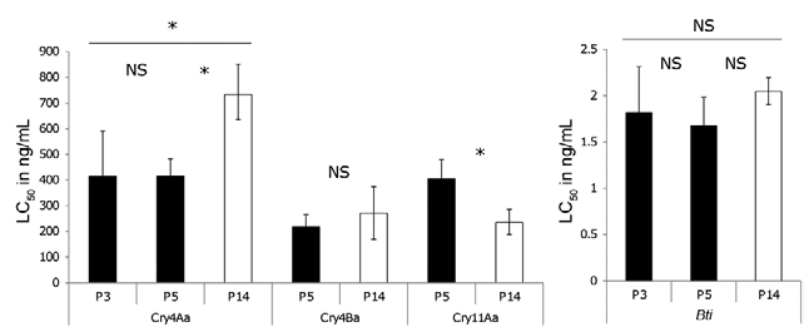

Fig. 2: lethal concentrations for $50 \%$ individuals $\left(\mathrm{LC}_{50}\right)$ of $\mathrm{P} 03, \mathrm{P} 05$ and P14 Aedes rusticus populations to Cry4Aa, Cry4Ba, Cry11Aa toxins and commercial Bacillus thuringiensis subsp. israelensis (Bti). Untreated populations are indicated in white and treated populations in black. Error: $95 \%$ confidence intervals calculated with the probit statistical analysis. 1160, 2320 and 2320 larvae were sampled from $\mathrm{P} 03, \mathrm{P} 05$ and $\mathrm{P} 14$ populations, respectively. Approximate distance between the sites: P03-P05, 70 km, P03-P14, 58 km and P05-P14, 85 km. Asterisks mean $\mathrm{p}<0.05$. NS: not significant. 

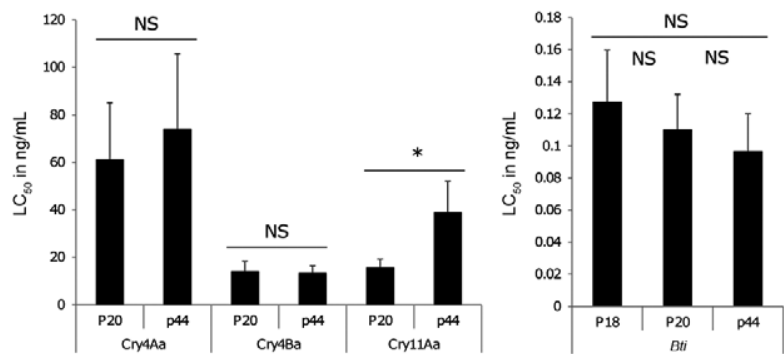

Fig. 3: lethal concentrations for $50 \%$ individuals $\left(\mathrm{LC}_{50}\right)$ of P18, P20 and $\mathrm{P} 44$ Aedes vexans populations to Cry4Aa, Cry4Ba, Cry11Aa toxins and commercial Bacillus thuringiensis subsp. israelensis (Bti). Error: $95 \%$ confidence intervals calculated with the probit statistical analysis. 380, 2004 and 2000 larvae were sampled from P18, P20 and $\mathrm{P} 44$ populations, respectively. Approximate distance between the sites: P18-P20, 21 km, P18-P44, 45 km and P20-P44, 66 km. Asterisks mean $\mathrm{p}<0.05$. NS: not significant.

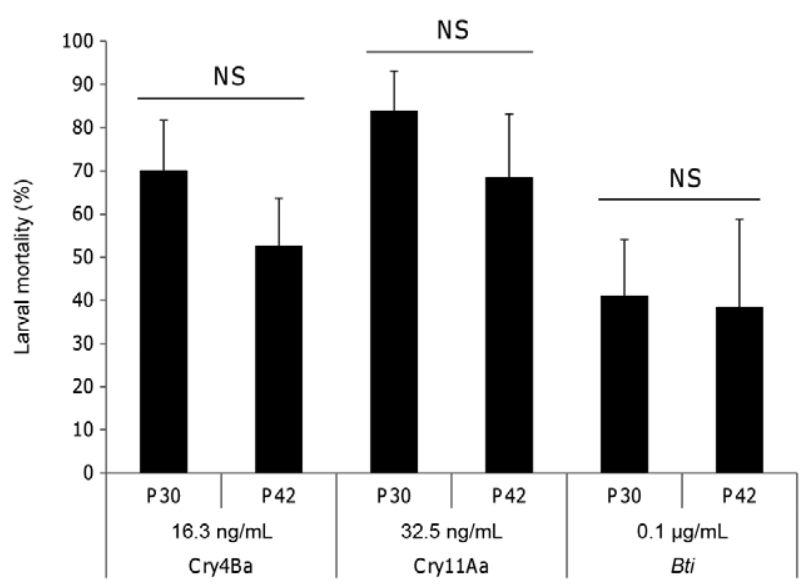

Fig. 4: differential mortality of $\mathrm{P} 30$ and $\mathrm{P} 42$ Aedes vexans populations to Cry4Ba (16.3 ng/mL), Cry11Aa (32.5 ng/mL) and commercial $B a$ cillus thuringiensis subsp. israelensis (Bti) $(0.1 \mu \mathrm{g} / \mathrm{mL})$. Error: standard error. 600 larvae were sampled from P30 and P42 populations. Approximate distance between the sites: P30-P42, 66 km. NS: not significant (Wilcoxon test).

Even if these changes were significant, tolerance ratios were low (less than 2.5-fold) possibly due to natural tolerance variations between populations rather than to an increased resistance selected by insecticide treatments.

The Ae. sticticus population P52 was significantly 3.5-fold and 8-fold more tolerant to Cry4Aa and Cry11Aa compared to P16 and P20 populations (mean of the two populations), while no significant difference was found with Bti (Fig. 5). This trend is similar to the resistance pattern observed for the resistant Aedes aegypti strain selected in the laboratory with field-persistent $B t i$ sampled in the Rhône-Alpes region which exhibited a moderate level of resistance to Bti (3.5-fold), but a higher resistance to individual Cry toxins (up to 60-fold) (Tetreau et al. 2012b). It is therefore possible that even if no difference of tolerance to $B t i$ was detectable in the three
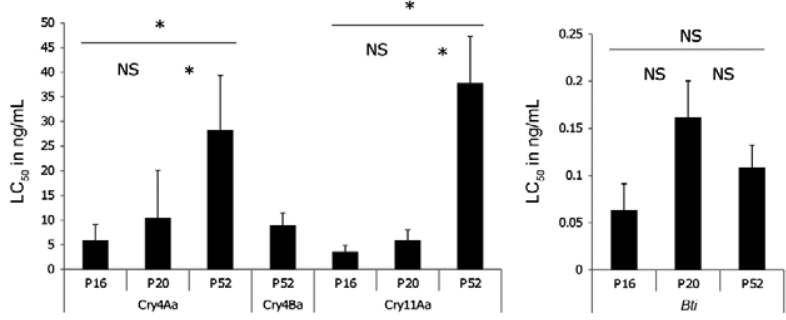

Fig. 5: lethal concentrations for 50\% individuals $\left(\mathrm{LC}_{50}\right)$ of P16, P20 and P52 Aedes sticticus populations to Cry4Aa, Cry4Ba, Cry11Aa toxins and commercial Bacillus thuringiensis subsp. israelensis (Bti). Error: $95 \%$ confidence intervals calculated with the probit statistical analysis. 1060, 1120 and 2280 larvae were sampled from P16, P20 and $\mathrm{P} 52$ populations, respectively. Approximate distance between the sites: P16-P20, 45 km, P16-P52, $58 \mathrm{~km}$ and P20-P52, $14 \mathrm{~km}$. Asterisks mean $\mathrm{p}<0.05$. NS: not significant.

populations of Ae. sticticus, a cryptic resistance may be developing in the P52 population, only detectable with bioassays using each Cry toxins separately. Moreover, these fluctuations could be partly explained by the environmental parameters in the collected sites (e.g. $\mathrm{pH}$ of the water, pollutants etc.) that could affect the susceptibility of the larvae, for example by inducing the production of detoxification enzymes or metabolites that may interfere with larval response to Bti toxins (Riaz et al. 2009, Poupardin et al. 2012). These effects were limited by keeping larvae $24 \mathrm{~h}$ in fresh water before performing the bioassays. This regularly $B t i$-sprayed breeding site was located behind a deserted windmill, close to both treated and untreated sites. No obvious characteristic, in terms of vegetation, ecological corridors and insecticide treatments strategy could explain the increased tolerance to $B t i$ toxins in this particular site. Regular bioassays with $B t i$ and individual toxins will confirm if tolerance of this population is increasing along with Bti treatments.

Toward a new diagnostic tool for Bti-resistance detection - Our results show that the tool developed in the present study is more sensitive than standard bioassays with Bti. The main limitation of its wide use is the low number of larvae sometime available in mosquito breeding sites. For this reason, the $\mathrm{LC}_{50}$ determination for each Cry toxin, even if it remains the best approach to precisely quantify the tolerance ratio between populations, does not seem well adapted to highly fragmented small and heterogeneous populations. Indeed, the diagnostic dose approach, which requires fewer toxin amount and fewer larvae, seems to be more adapted in order to gather tolerance data for a large number of population in a reasonable time. Based on the present study, empirical diagnostic doses can be estimated for various species studied and can be used for further studies aiming at monitoring Bti resistance in natural mosquito populations. Considering that previous works correlated a low level of resistance to Bti with high level of resistance to individual Cry toxins (Paris et al. 2011, Tetreau et al. 2012b), it is expected that bioassays with Cry toxins would detect an incipient resistance to Bti before it 
could be detected with classical bioassays with $B t i$. This hypothesis has to be confirmed by monitoring the evolution of the tolerance of the populations tested in this study for several years. In combination with bioassays with $B t i$, bioassays with separate Cry toxins allow a more sensitive monitoring of the susceptibility of fieldmosquito populations to Bti. Therefore, we believe that such approach should be used in further studies looking for $B t i$-resistance in other species from the Rhône-Alpes region (e.g. Ae. cantans), from the Mediterranean region (e.g. Aedes detritus, Aedes caspius) and should also be applied to human pathogens vector species such as $A e$. aegypti, Aedes albopictus and Cx. pipiens exposed to regular Bti treatments.

$B t i$ is widely used for mosquito control. Based on observations of the resistance phenotype of a Bti-resistant laboratory strain, we developed a new tool: bioassays with individual Bti Cry toxins. This highly sensitive tool could be used in combination with bioassays with $B t i$ in order to improve the monitoring of the susceptibility of fieldpopulations of mosquitoes regularly treated with Bti.

\section{ACKNOWLEDGEMENTS}

To Brian Federici, for providing recombinant $B t$ strains, to the staff of the mosquito control agency EID, for precious help in mosquito sampling and species identification, to Thierno Mamadou Diallo and undergraduate students, for their help for bioassays, and to the anonymous reviewers, for constructive comments.

\section{REFERENCES}

Akiner MM, Simsek FM, Caglar SS 2009. Insecticide resistance of Culex pipiens (Diptera: Culicidae) in Turkey. J Pestic Sci 34: 259-264.

Alcantara E, Estrada A, Alpuerto V, Head G 2011. Monitoring Cry1$\mathrm{Ab}$ susceptibility in Asian corn borer (Lepidoptera: Crambidae) on Bt corn in the Philippines. Crop Prot 30: 554-559.

Becker N, Peric D, Zgomba M, Boase C, Dahl C, Madon M, Kaiser A 2010. Mosquitoes and their control, Kluwer Academic/Plenum Publishers, New York, 518 pp.

Boyer S, Paris M, Jego S, Lemperiere G, Ravanel P 2012. Influence of insecticide Bacillus thuringiensis subsp. israelensis treatments on resistance and enzyme activities in Aedes rusticus larvae (Diptera: Culicidae). Biol Control 62: 75-81.

Boyer S, Tilquin M, Ravanel P 2007. Differential sensitivity to Bacillus thuringiensis var. israelensis and temephos in field mosquito populations of Ochlerotatus cataphylla (Diptera: Culicidae): Toward resistance? Environ Toxicol Chem 26: 157-162.

Carriere Y, Crowder DW, Tabashnik BE 2010. Evolutionary ecology of insect adaptation to Bt crops. Evol Appl 3: 561-573.

Gao YL, An JJ, Liu CX, Wu KM 2010. Monitoring of Bt resistance from field-collected Helicoverpa armigera populations in Northern China. Southwest Entomol 35: 399-402.

Gassmann AJ, Petzold-Maxwell JL, Keweshan RS, Dunbar MW 2011. Field-evolved resistance to $B t$ maize by Western corn rootworm. PLOS ONE 6: e22629.

Janmaat AF, Myers J 2003. Rapid evolution and the cost of resistance to Bacillus thuringiensis in greenhouse populations of cabbage loopers, Trichoplusia ni. Proc R Soc Lond B Biol Sci 270: 2263-2270.

Kamgang B, Marcombe S, Chandre F, Nchoutpouen E, Nwane P, Etang J, Corbel V, Paupy C 2011. Insecticide susceptibility of
Aedes aegypti and Aedes albopictus in Central Africa. Parasit Vectors 4: 79.

Liu H, Cupp EW, Guo AG, Liu NN 2004. Insecticide resistance in Alabama and Florida mosquito strains of Aedes albopictus. J Med Entomol 41: 946-952.

Loke SR, Andy-Tan WA, Benjamin S, Lee HL, Sofian-Azirun M 2010. Susceptibility of field-collected Aedes aegypti (L.) (Diptera: Culicidae) to Bacillus thuringiensis israelensis and temephos. Trop Biomed 27: 493-503.

Marcombe S, Poupardin R, Darriet F, Reynaud S, Bonnet J, Strode C, Brengues C, Yebakima A, Ranson H, Corbel V, David JP 2009. Exploring the molecular basis of insecticide resistance in the dengue vector Aedes aegypti: a case study in Martinique Island (French West Indies). BMC Genomics 10: 494.

Medlock JM, Hansford KM, Schaffner F, Versteirt V, Hendrickx G, Zeller H, Van Bortel W 2012. A review of the invasive mosquitoes in Europe: ecology, public health risks, and control options. Vector Borne Zoonotic Dis 12: 435-447.

Meissle M, Romeis J, Bigler F 2011. Bt maize and integrated pest management - a European perspective. Pest Manag Sci 67: 1049-1058.

Ocampo CB, Salazar-Terreros MJ, Mina NJ, McAllister J, Brogdon W 2011. Insecticide resistance status of Aedes aegypti in 10 localities in Colombia. Acta Trop 118: 37-44.

Paris M, Boyer S, Bonin A, Collado A, David JP, Despres L 2010. Genome scan in the mosquito Aedes rusticus: population structure and detection of positive selection after insecticide treatment. Mol Ecol 19: 325-337.

Paris M, Tetreau G, Laurent F, Lelu M, Despres L, David J-P 2011. Persistence of Bacillus thuringiensis israelensis (Bti) in the environment induces resistance to multiple Bti toxins in mosquitoes. Pest Manag Sci 67: 122-128.

Paul A, Harrington LC, Zhang L, Scott JG 2005. Insecticide resistance in Culex pipiens from New York. J Am Mosq Control Assoc 21: 305-309.

Poupardin R, Riaz MA, Jones CM, Chandor-Proust A, Reynaud S, David JP 2012. Do pollutants affect insecticide-driven gene selection in mosquitoes? Experimental evidence from transcriptomics. Aquat Toxicol 114-115: 49-57.

R Development Core Team 2007. R: a language and environment for statistical computing. Available from: R-project.org.

Riaz MA, Poupardin R, Reynaud S, Strode C, Ranson H, David JP 2009. Impact of glyphosate and benzo[a]pyrene on the tolerance of mosquito larvae to chemical insecticides. Role of detoxification genes in response to xenobiotics. Aquat Toxicol 93: 61-69.

Rivero A, Vezilier J, Weill M, Read AF, Gandon S 2010. Insecticide control of vector-borne diseases: when is insecticide resistance a problem? PLoS Pathog 6: e1001000.

Savary S, Horgan F, Willocquet L, Heong KL 2012. A review of principles for sustainable pest management in rice. Crop Prot 32: 54-63.

Shin EH, Park C, Kim HK, Lee DK, Kim SI, Kang H, Chang KS 2011. Insecticide susceptibility of Ephemera orientalis (Ephemeroptera: Ephemeridae) and two mosquito species, Anopheles sinensis and Culex pipiens in the Republic of Korea. J Asia Pac Entomol 14: 233-236.

Silva M, de Melo Chalegre KD, Anastacio DB, de Oliveira CMF, da Silva SB, Acioli RV, Hibi S, de Oliveira DC, Parodi ESM, Marques CAM, Furtado AF, Regis L 2008. Culex quinquefasciatus field populations subjected to treatment with Bacillus sphaericus did not display high resistance levels. Biol Control 44: 227-234.

Silva TBM, Siqueira HAA, Oliveira AC, Torres JB, Oliveira JV, Montarroyos PAV, Farias M 2011. Insecticide resistance in Brazilian 
populations of the cotton leaf worm, Alabama argillacea. Crop Prot 30: 1156-1161.

Sivasupramaniam S, Head GP, English L, Li YJ, Vaughn TT 2007. A global approach to resistance monitoring. J Invertebr Pathol 95: 224-226.

Springate S, Colvin J 2012. Pyrethroid insecticide resistance in British populations of the cabbage whitefly, Aleyrodes proletella. Pest Manag Sci 68: 260-267.

Su TY, Mulla MS 2004. Documentation of high-level Bacillus sphaericus 2362 resistance in field populations of Culex quinquefasciatus breeding in polluted water in Thailand. J Am Mosq Control Assoc 20: 405-411.

Tabashnik BE, Biggs RW, Fabrick JA, Gassmann AJ, Dennehy TJ, Carriere Y, Morin S 2006. High-level resistance to Bacillus thuringiensis toxin CrylAc and cadherin genotype in pink bollworm. J Econ Entomol 99: 2125-2131.

Tabashnik BE, Carriere Y 2010. Field-evolved resistance to Bt cotton bollworm in the US and pink bollworm in India. Southwest Entomol 35: 417-424.

Tabashnik BE, Cushing NL, Finson N, Johnson MW 1990. Field development of resistance to Bacillus-thuringiensis in diamondback moth (Lepidoptera, Plutellidae). J Econ Entomol 83: 1671-1676.

Tabashnik BE, Gassmann AJ, Crowder DW, Carriere Y 2008. Insect resistance to Bt crops: evidence versus theory. Nat Biotechnol 26: 199-202.

Tetreau G, Alessi M, Veyrenc S, Périgon S, David JP, Reynaud S, Després L 2012a. Fate of Bacillus thuringiensis subsp. israelensis in the field: evidence for spore recycling and differential persistence of toxins in leaf litter. Appl Environ Microbiol 78: 8362-8367.

Tetreau G, Bayyareddy K, Jones CM, Stalinski R, Riaz MA, Paris M, David JP, Adang MJ, Despres L 2012b. Larval midgut modifications associated with Bti resistance in the yellow fever mosquito using proteomic and transcriptomic approaches. $B M C$ Genomics 13: 248.

Tilquin M, Paris M, Reynaud S, Despres L, Ravanel P, Geremia RA, Gury J 2008. Long lasting persistence of Bacillus thuringiensis subsp. israelensis (Bti) in mosquito natural habitats. PLoS ONE 3: e3432.

van den Berg H 2011. Global status of DDT and its alternatives for use in vector control to prevent disease. Cien Saude Colet 16: 575-590.

van Griensven J, Diro E 2012. Visceral leishmaniasis. Infect Dis Clin North Am 26: 309-322.

Vasquez MI, Violaris M, Hadjivassilis A, Wirth MC 2009. Susceptibility of Culex pipiens (Diptera: Culicidae) field populations in Cyprus to conventional organic insecticides, Bacillus thuringiensis subsp. israelensis and methoprene. J Med Entomol 46: 881-887.

WHO - World Health Organization 2005. Guidelines for laboratory and field testing of mosquito larvicides, WHO, Geneva, $39 \mathrm{pp}$.

Zhang HY, Yang CJ, Huang JY, Lu L 2004. Susceptibility of field populations of Anopheles sinensis (Diptera: Culicidae) to Bacillus thuringiensis subsp. israelensis. Biocontrol Sci Technol 14: 321-325. 

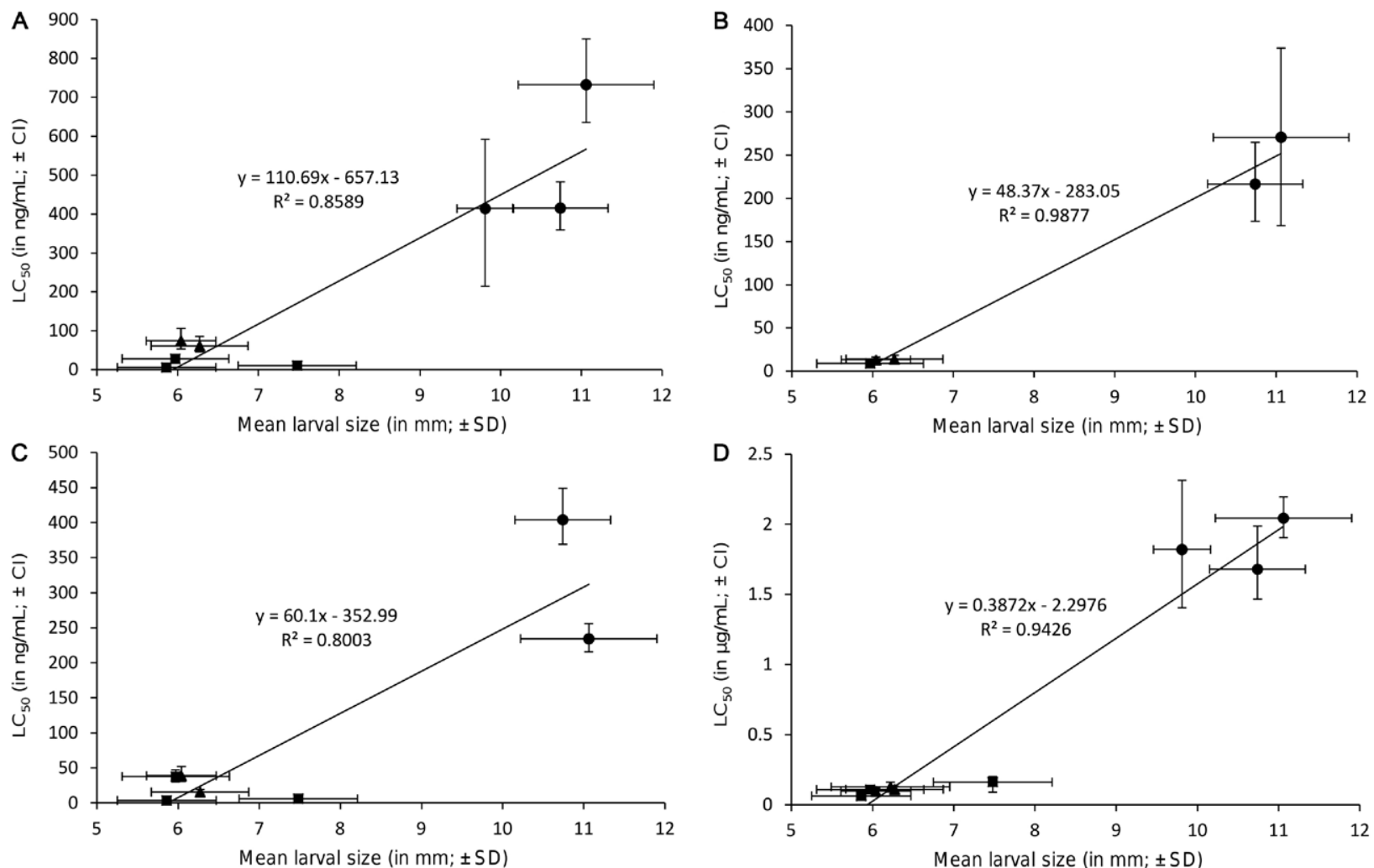

Lethal concentration for $50 \%$ of individuals $\left(\mathrm{LC}_{50}\right.$ ) of Aedes rusticus (circle), Aedes sticticus (square) and Aedes vexans (triangle) populations for Bacillus thuringiensis subsp. israelensis (Bti) toxins Cry4Aa (A), Cry4Ba (B), Cry11 Aa (C) and commercial Bti (D) in function of the mean larval size (in $\mathrm{mm}$ ) per population. $\mathrm{LC}_{50}$ and their $95 \%$ confidence intervals $(\mathrm{CI}$ ) were calculated using a Probit regression. Errors for the larval size represent standard deviation (SD) of mean. 